Ground st at e correl at i ons of the quant um Toda I at ti ce

\begin{tabular}{|l|l|}
\hline 著者 & Nat suyana Aki hi ko \\
\hline $\begin{array}{l}\text { j ournal or } \\
\text { publ i cat i on ti t l e }\end{array}$ & Physi cs Let ter s A \\
\hline vol une & 228 \\
\hline nunber & $1-2$ \\
\hline page r ange & $79-83$ \\
\hline year & $1997-03$ - 31 \\
\hline 出版者 & El sevi er \\
\hline 権利 & (c) 1997 Publ i shed by El sevi er Sci ence B. V. \\
\hline URL & ht t p: //hdl . handl e. net /10297/618 \\
\hline
\end{tabular}




\title{
Ground state correlations of the quantum Toda lattice
}

\author{
A. Matsuyama \\ Department of Physics, Faculty of Science, Shizuoka University, 836 Ohya, Shizuoka 422, Japan
}

\begin{abstract}
Based on the Bethe ansatz equation and the finite-size scaling analysis of conformal field theory, we calculate critical exponents of the ground state correlations of the quantum Toda lattice. It is shown that the Toda lattice belongs to the $c=1$ Gaussian universality class.
\end{abstract}

PACS numbers: 05.50.+q, 05.70.Jk, 11.25.Hf, 64.60.Fr

Keywords: Toda lattice, Bethe ansatz, Correlation function, Conformal field theory 
There has been considerable progress in understanding critical properties of onedimensional integrable models by combing Bethe ansatz method and conformal field theory (CFT) [1]. Many lattice models (Heisenberg model, Hubbard model and supersymmetric $t-J$ model [2] etc.) and continuum models (nonlinear Schrödinger model and CalogeroSutherland model $[3,4]$ etc.) have been studied in a systematic way [5-10]. Most of these models have a critical point at $T=0$ and excitations are gapless. Their correlation functions decay asymptotically as powers of the distance. It is now recognized that they belong to the same universality class described by the $c=1$ Gaussian CFT.

The Toda lattice is also one of the most popular integrable models and has been studied extensively over two decades [11]. It consists of many particles interacting with a nearestneighbour potential of an exponential type. The classical equations of motion can be given in a Lax form [12] and thus conserved quantities are easily constructed. These conserved quantities are of Hénon-type [13] and, in the quantization procedure, ordering problems of operators $\left\{\hat{x}_{i}, \hat{p}_{i}\right\}$ do not occur. A systematic way of the quantization of the periodic Toda lattice has been developed by Gutzwiller [14]. He succeeded in constructing simultaneous eigenfunctions of the operators of conserved quantities for $N=2,3$ and 4 particle lattices. Later, his method was extended generally for $N$-particle Toda lattice by Pasquier and Gaudin [15]. Those quantization conditions are rather complicated and they are not in the form of the Bethe ansatz equation. Sklyanin [16] has combined the quantum spectral transform method (QSTM) (R-matrix formalism) and Gutzwiller's formulation and derived an equation for the spectrum of the quantum Toda lattice. Fowler and Frahm [17] studied the periodic Toda lattice based on Gutzwiller's quantization conditions and pointed out that the energy spectra of a finite particle system are different from those given by Bethe ansatz equation. Recently we have shown $[18,19]$ that the quantization conditions are significantly simplified in the large $N$ limit and they become ordinary forms of the Bethe ansatz equations. In Ref. $[18,19]$ we have studied bulk properties of the Toda lattice, i.e., ground state energy and thermodynamics. They are found to be essentially the same as those given by Hader and Mertens [20] who assumed asymptotic Bethe ansatz for the Toda lattice. How- 
ever, correlation functions and critical properties have not been investigated so far. In this Letter, we will show that critical properties of the quantum Toda lattice can also be described based on the finite-size scaling analysis of CFT and it belongs to the $c=1$ Gaussian universality class.

The Hamiltonian of the $N$-particle Toda lattice confined in a box with length $L$ is given in a dimensionless form as [19]

$$
H=-\frac{\hbar^{2}}{2} \sum_{i=1}^{N} \frac{\partial^{2}}{\partial x_{i}^{2}}+e^{-L / N} \sum_{i=1}^{N} \exp \left(x_{i}-x_{i+1}\right)
$$

where we set a periodic boundary condition $x_{N+1}=x_{1}$. Since the interactions are of nearestneighbour type, particles are neither bosons nor fermions. This is a distinctive feature of the Toda lattice which contrasts with other continuum models. Instead the system has the symmetry of the dihedral group $D_{N}$ and eigenstates can be classified according to the irreducible representations of the $D_{N}$ group [21]. In Eq.(1) we have rescaled the constant $\hbar$, Plank's constant divided by $2 \pi$, as $\hbar /\left(m a^{2} V_{0}\right) \rightarrow \hbar$, where $m$ is the mass of a particle, $V_{0}$ and $a$ are the strength and the range of the potential respectively. Since the Toda lattice is not scale-invariant, $\hbar$ sets the scale of the system. We will write $\hbar$ explicitly since we will discuss the scale dependence of the energy and critical exponents. The Hamiltonian is the same as that of a periodic Toda lattice and we will apply quantization conditions given by Gutzwiller [14], which are rather involved and we will not review them here. In Ref. [18,19] we have shown that they are largely simplified in the large $N$ limit and become

$$
2 \pi n_{i}=L k_{i}+2 N k_{i} \ln \hbar+2 \sum_{j} \arg \left(\Gamma\left(1+i\left(k_{i}-k_{j}\right)\right)\right)
$$

where $n_{i}$ is an integer (a half-odd integer) for $N=$ odd (even) and $k_{i}^{\prime} s$ are rapidities. Eq.(2) is valid for the system with total momentum $P=\hbar \sum_{j} k_{j}=0$ and only intrinsic degrees of freedom are taken into account. However, we will also consider the current excitaion which shifts the center of mass without changing internal motions. Taking into account the current excitaion, Eq.(2) should be modified to

$$
2 \pi n_{i}=L k_{i}+2 N k_{i} \ln \hbar+2 \sum_{j} \arg \left(\Gamma\left(1+i\left(k_{i}-k_{j}\right)\right)\right)-2 \sum_{j} k_{j} \ln \hbar
$$




$$
=L k_{i}+2 \sum_{j}\left(\left(k_{i}-k_{j}\right) \ln \hbar+\arg \left(\Gamma\left(1+i\left(k_{i}-k_{j}\right)\right)\right)\right) .
$$

Eq.(3) is an ordinary form of the Bethe ansatz equation. The momentum and the energy of the system are

$$
P=\frac{2 \pi \hbar}{L} \sum_{j} n_{j}=\hbar \sum_{j} k_{j}, \quad E=\frac{\hbar^{2}}{2} \sum_{j} k_{j}^{2} .
$$

In the thermodynamic limit $(N, L \rightarrow \infty$, with the density $D=N / L$ kept constant), the rapidities $\left\{k_{i}\right\}$ in the ground state are distributed in a finite interval $[-Q, Q]$ with a distribution function $\rho(k)$ satisfying

$$
\rho(x)=\frac{1}{2 \pi}+\int_{-Q}^{Q} \frac{d y}{2 \pi} K(x-y) \rho(y)
$$

where the kernel is $K(x-y)=2(\ln \hbar+\Re \psi(1+i(x-y))), \psi(z)$ being the digamma function, i.e., $\psi(z)=\Gamma^{\prime}(z) / \Gamma(z)$. The density distribution $\rho(x)$ is normalized as $\int_{-Q}^{Q} \rho(x) d x=N / L=$ $D$. The energy per particle is

$$
E / N=D^{-1} \hbar^{2} \int_{-Q}^{Q} \frac{1}{2} x^{2} \rho(x) d x
$$

The dressed energy $\varepsilon(k)$ of an elementary excitation is given by the integral equation

$$
\varepsilon(x)=\varepsilon_{0}(x)+\int_{-Q}^{Q} \frac{d y}{2 \pi} K(x-y) \varepsilon(y)
$$

where $\varepsilon_{0}(k)$ is the bare energy, i.e., $\varepsilon_{0}(k)=\hbar^{2} k^{2} / 2-\mu$ and the chemical potential $\mu$ is determined by the condition $\varepsilon( \pm Q)=0$.

The kernel of the integral equations $(5,7)$ is quite different from those for the Heisenberg model and nonlinear Schrödinger model which have Lorenzian-type kernels. The function $K(x)=2(\ln \hbar+\Re \psi(1+i x))$ is an even function and monotonically increases for $x>0$. At $x=0, K(0)=2\left(\ln \hbar-\gamma_{E}\right), \gamma_{E}$ being Euler's constant and $K(x) \sim 2 \ln (\hbar x)$ for $x \rightarrow$ $\infty$. The maximum eigenvalue of the kernel becomes unity at a certain critical value $Q_{c}$ where the density diverges $D \rightarrow \infty$. For example, for the scales $\hbar=10^{-2}, 1,10^{2}, Q_{c} \simeq$ 207.3, 2.899, 0.3807 respectively and $\partial Q_{c} / \partial \hbar<0$. In the interval $0<Q<Q_{c}$, the density $D$ is a monotonically increasing function of $Q$, i.e., $\partial D / \partial Q>0$. Since Eq.(5) is rewritten as 


$$
\rho(x)=\frac{1}{\pi} D \ln \left(\hbar e^{1 / 2 D}\right)+\frac{1}{\pi} \int_{-Q}^{Q} d y \Re \psi(1+i(x-y)) \rho(y)
$$

the functional form is determined only by $Q$ apart from a normalization and thus the combination $\hbar e^{1 / 2 D}$ is also a function of $Q$. Therefore the energy per particle $u=E / N$ is also a function of $Q$. Our numerical calculation gives an empirical form $u \sim \hbar^{2} \times C Q^{\alpha}$ with $C \simeq 0.177, \alpha \simeq 2.11$ for the wide range $10^{-2}<Q<10^{2}$.

In Fig.1 we show the energy per particle $u$ as a function of the density $D . \quad u$ is monotonically increasing and, at $D=\infty$, it coincides a chemical potential $\mu$, since $\mu N=G=E-T S+P L=E$ at $T=0, L=0$. In the case $\hbar \rightarrow \infty\left(Q_{c} \rightarrow 0\right)$ and low density $D \ll 1$, the range of the potential becomes effectively very small and it becomes a hard-wall potential, i.e., $V(x)=0(\infty)$ for $x<0(x>0)$. The particles interacting with a hard-wall potential behave in the same way as hard-core bosons (bosons interacting with an infinitely repulsive $\delta$-function potential) or free spinless fermions. The energy per particle $u$ of the free Fermi gas is $u=\pi^{2} \hbar^{2} D^{2} / 6$ and it is also shown in Fig. 1 for the scale $\hbar=10^{2}$. On the other hand, the case $\hbar \rightarrow 0\left(Q_{c} \rightarrow \infty\right)$ corresponds to a classical system. The classical ground state is a static configuration of particles and rapidity $k_{j}$ is given by $k_{j}=Q t_{j}$ where $t_{j}=\cos \pi(2 j+1) / 2 N(j=0,1, \ldots, N-1)$ is a zero of Chebyshev's polynomial. Thus the density distribution becomes $\rho(x)=D\left(Q^{2}-x^{2}\right)^{-1 / 2} / \pi$, which is an approximate solution of Eq.(5) given by Sutherland [22]. Employing the solution $\rho(x)=D\left(Q^{2}-x^{2}\right)^{-1 / 2} / \pi$, one can obtain $Q \simeq 2 \hbar^{-1} e^{-1 / 2 D}$ and $u \simeq \hbar^{2} Q^{2} / 4 \simeq e^{-1 / D}$, which coincides an energy per particle of the classical system. The classical energy density $u=e^{-1 / D}$ is also shown in Fig.1.

Let us apply the finite-size scaling analysis to the Toda lattice. The central charge $c$ of Virasoro algebra can be easily extracted from the finite-size correction to the ground state energy $E_{0}[23,24]$

$$
E_{0}=L \varepsilon_{\infty}-\frac{\pi v}{6 L}
$$

where $\varepsilon_{\infty}$ is the energy density of the infinite system and $v=\varepsilon^{\prime}(Q) /(2 \pi \rho(Q))$ is the Fermi velocity. From Eq.(9) it is readily recognized that the Toda lattice can be described by 
the $c=1$ Gaussian CFT. The central charge $c=1$ can also be obtained from the low temperature behaviour of the specific heat $C \simeq \pi T / 3 v$ which was calculated by Yang-Yang's [25] thermodynamic formulation of the Toda lattice [19].

Next we will examine a tower structure of the energy spectrum [26]. Low energy excitations are characterized by (a) the change of particle number $\Delta N$, (b) the backscattering of $\Delta D$ particles from one Fermi point to other one and (c) $N^{ \pm}$particle-hole excitations at Fermi boundaries. The energy and the momentum of these excitations are expressed as

$$
\begin{aligned}
& E-E_{0}=\frac{2 \pi v}{L}\left(\frac{1}{4 Z^{2}} \Delta N^{2}+Z^{2} \Delta D^{2}+N^{+}+N^{-}\right) \\
& P-P_{0}=\frac{2 \pi \hbar}{L}\left(\Delta N \Delta D+N^{+}-N^{-}\right)+2 p_{F} \Delta D
\end{aligned}
$$

where $p_{F}=\pi \hbar N / L$ is the Fermi momentum. $Z$ is the dressed charge given by $Z=\xi( \pm Q)$, where the function $\xi(x)$ is defined by the integral equation

$$
\xi(x)=1+\int_{-Q}^{Q} \frac{d y}{2 \pi} K(x-y) \xi(y)
$$

and its solution is $\xi(x)=2 \pi \rho(x)$. From Eqs. $(10,11)$ we can assign the conformal dimensions $\Delta^{ \pm}$of the $c=1$ Gaussian CFT as

$$
\Delta^{ \pm}=\frac{1}{2}\left(\frac{\Delta N}{2 Z} \pm Z \Delta D\right)^{2}+N^{ \pm} .
$$

It is now straightforward to obtain a long-distance behaviour of correlation functions. The asymptotic form of the density-density correlation function is given by [6]

$$
\langle n(x) n(0)\rangle \sim D^{2}\left(1-\theta(2 \pi D x)^{-2}+A(D x)^{-\theta} \cos 2 \pi D x+\cdots\right)
$$

where $n(x)$ is the density operator and $\langle n(x)\rangle=D$. The critical exponent $\theta$ of $2 p_{F}=2 \pi \hbar D$ oscillation term is given by the excitaion $\left(\Delta N, \Delta D, N^{ \pm}\right)=(0,1,0)$ and $\theta=2\left(\Delta^{+}+\Delta^{-}\right)=$ $2 Z^{2}$.

In Fig. 2 we show the critical exponent $\theta$ as a function of the density $D$. In the case of low density limit $D \rightarrow 0$, the critical exponent $\theta$ approaches to 2 , while for the limit $D \rightarrow \infty$, $\theta \rightarrow \infty$ irrespective of the scale $\hbar$. In the low density region $D \ll 1$, the critical exponent $\theta$ is expressed as 


$$
\theta \simeq 2\left(1+4 D\left(\ln \hbar-\gamma_{E}\right)\right)
$$

Therefore, for $\hbar>e^{\gamma_{E}} \simeq 1.78, \theta$ is monotonically increasing with $D$, while for $\hbar<e^{\gamma_{E}}$, $(\partial \theta / \partial D)_{D=0}<0$ and $\theta$ has a minimum. The system with $\hbar \gg 1$ and $D \ll 1$ corresponds to that of the hard-wall potential and particles behave like hard-core bosons, while for $\hbar \ll 1$ and $D \ll 1$ it reduces to free particles since the exponential potential becomes flat. The case of $\hbar \rightarrow 0$ with a finite density $D$ corresponds to a classical system and $\theta \rightarrow 0$. The classical ground state is a static configuration of particles with an equal spacing $1 / D$ and its correlation function is

$$
\begin{aligned}
\langle n(x) n(0)\rangle & =\sum_{l} D^{2} \delta(D x-l) \\
& =D^{2}(1+2 \cos 2 \pi D x+2 \cos 4 \pi D x+\cdots)
\end{aligned}
$$

and the correlation does not decay, which results in $\theta=0$. Fig.2 shows that there are points of $\theta=1$ for the scales $\hbar \lesssim 0.4$. At these points, the dressed charge is $Z=1 / \sqrt{2}$ and the $\mathrm{U}(1)$ symmetry of the system is enhanced to that of level-1 SU(2) current algebra. The behaviour of the critical exponent $\theta$ is quite different from those of other continuum models. For example, nonlinear Schrödinger model is scale-invariant, i.e., a change of the strength $\kappa$ of the $\delta$-function interaction is equivalent to the change of the length scale, thus $\theta$ is a function of $\kappa D=\kappa N / L$, and $\theta>2$ for any value of $\kappa D$. On the other hand, CalogeroSutherland model is not scale-invariant, however, the critical exponent $\theta$ depends only on the strength of the interaction and does not depend on the density [10].

Next let us consider the asymptotic behaviour of the field correlator $\left\langle\psi(x, t) \psi^{\dagger}(0,0)\right\rangle$. In this case the leading term can be obtained by assigning $\left(\Delta N, \Delta D, N^{ \pm}\right)=(1,0,0)$ and it is

$$
\left\langle\psi(x, t) \psi^{\dagger}(0,0)\right\rangle \sim B|x+i v t|^{-\eta}
$$

with $\eta=1 /\left(2 Z^{2}\right)=1 / \theta$. This scaling relation is the same as 1 -dimensional Bose gas.

In summary, we have shown that the quantum Toda lattice belongs to the $c=1$ Gaussian universality class of CFT and low energy excitations show a tower structure of the CFT energy spectra. For the scale $\hbar \rightarrow \infty$ and $D \ll 1$, which corresponds to the hard-wall 
potential, the system behaves like hard-core bosons and the critical exponent is $\theta=2$, while the case $\hbar \rightarrow 0$ corresponds to a classical system where particles are localized with an equal spacing and the critical exponent vanishes. 


\section{REFERENCES}

[1] A.A. Belavin, A.M. Polyakov and A.B. Zamolodchikov, Nucl.Phys. B241, 333 (1984)

[2] P. Schlottmann, Phys.Rev. B 36, 5177 (1987)

[3] F. Calogero, J.Math.Phys. 10, 2191 (1969); 12, 419 (1971)

[4] B. Sutherland, Phys.Rev. A 4, 2019 (1971); A 5 , 1372 (1972)

[5] V.E. Korepin, N.M. Bogoliubov and A.G. Izergin, Quantum Inverse Scattering Method and Correlation Functions (Cambridge, 1993)

[6] N.M. Bogoliubov, A.G. Izergin and V.E. Korepin, Nucl.Phys. B275, 687 (1986)

[7] F. Woynarovich, J.Phys. A 22, 4243 (1989)

[8] H. Frahm and V.E. Korepin, Phys.Rev. B 42, 10553 (1990)

[9] N. Kawakami and S.-K. Yang, Phys.Rev. Lett. 65, 2309 (1990); J.Phys. Condens. Matter 35983 (1991)

[10] N. Kawakami and S.-K. Yang, Phys.Rev. Lett. 67, 2493 (1991)

[11] M. Toda, J.Phys.Soc.Japan 22, 431 (1969); Theory of nonlinear lattices (Springer, Berlin, 1981)

[12] H. Flaschka, Phys.Rev. B 9, 1924 (1974)

[13] M. Hénon, Phys.Rev. B 9, 1921 (1974)

[14] M.C. Gutzwiller, Ann.Phys. (NY) 124, 347 (1980); 133, 304 (1981)

[15] V. Pasquier and M. Gaudin, J.Phys. A 25, 5243 (1992)

[16] E.K. Sklyanin, in Lecture Notes in Physics, Vol.226 (Springer, Berlin, 1985)

[17] M. Fowler and H. Frahm, Phys.Rev. B 39, 11800 (1989)

[18] A. Matsuyama, J.Phys. A 29, 1089 (1996) 
[19] A. Matsuyama, J.Phys. A 29, 4837 (1996)

[20] M. Hader and F.G. Mertens, J.Phys. A 19, 1913 (1986)

[21] A. Matsuyama, Ann.Phys. (NY) 220, 300 (1992)

[22] B. Sutherland, Rocky Mount.J.Math. 8, 413 (1978)

[23] H.W. Blöte, J.L. Cardy and M.P. Nightingale, Phys.Rev. Lett. 56, 742 (1986)

[24] I. Affleck, Phys.Rev. Lett. 56, 746 (1986)

[25] C.N. Yang and C.P. Yang, J.Math.Phys. 10, 1115 (1969)

[26] J.L. Cardy, Nucl.Phys. B270, 186 (1986) 


\section{FIGURES}

FIG. 1. Energy density $u=E / N$ versus $D$ for scales $\hbar=10^{2}, 10,1$ and $10^{-1}$. Energy densities of the classical value $u=e^{-1 / D}$ and free Fermi gas $u=\pi^{2} \hbar^{2} D^{2} / 6\left(\hbar=10^{2}\right)$ are also shown by dashed and dot-dashed lines respectively.

FIG. 2. Critical exponent $\theta$ versus $D$ for scales $\hbar=10^{2}, 10,1,10^{-1}$ and $10^{-2}$. 